\title{
The endoplasmic reticulum-associated mRNA-binding proteins ERBP1 and ERBP2 interact in bloodstream-form Trypanosoma brucei
}

\author{
Kathrin Bajak ${ }^{1,2}$, Kevin Leiss ${ }^{1}$, Christine Clayton ${ }^{1}$, Esteban Erben ${ }^{\text {Corresp. } 2}$ \\ ${ }^{1}$ Zentrum für Molekulare Biologie der Universität Heidelberg (ZMBH), Heidelberg, Germany \\ 2 Deutsches Krebsforschungszentrum (DKFZ), Heidelberg, Germany \\ Corresponding Author: Esteban Erben \\ Email address: e.erben@dkfz-heidelberg.de
}

Kinetoplastids rely heavily on post-transcriptional mechanisms for control of gene expression, and on RNA-binding proteins that regulate mRNA splicing, translation and decay. Trypanosoma brucei ERBP1 (Tb927.10.14150) and ERBP2 (Tb927.9.9550) were previously identified as mRNA binding proteins that lack canonical RNA-binding domains. We here show that ERBP1 is associated with the endoplasmic reticulum, like ERBP2, and that the two proteins interact in vivo. Loss of ERBP1 from bloodstream-form T. brucei initially resulted in a growth defect but proliferation was restored after more prolonged cultivation. Pull-down analysis of tagged ERBP1 suggest that it preferentially binds to ribosomal protein mRNAs. The ERBP1 sequence resembles that of Saccharomyces cerevisiae Bfr1, which also localises to the endoplasmic reticulum and binds to ribosomal protein mRNAs. However, unlike Bfr1, ERBP1 does not bind to mRNAs encoding secreted proteins, and it is also not recruited to stress granules after starvation. 
1 The endoplasmic reticulum-associated mRNA-binding proteins ERBP1 and ERBP2

2 interact in bloodstream-form Trypanosoma brucei.

3 Kathrin Bajak ${ }^{a, b}$, Kevin Leiss ${ }^{a}$, Christine Clayton ${ }^{a}$ and *Esteban Erben ${ }^{b}$

4

aZentrum für Molekulare Biologie der Universität Heidelberg (ZMBH), Heidelberg, Germany.

bDeutsches Krebsforschungszentrum (DKFZ), Heidelberg, Germany.

* Corresponding author: e.erben@dkfz-heidelberg.de

\begin{abstract}
9 Abstract
10 Kinetoplastids rely heavily on post-transcriptional mechanisms for control of gene expression, and 11 on RNA-binding proteins that regulate mRNA splicing, translation and decay. Trypanosoma brucei 12 ERBP1 (Tb927.10.14150) and ERBP2 (Tb927.9.9550) were previously identified as mRNA 13 binding proteins that lack canonical RNA-binding domains. We here show that ERBP1 is 14 associated with the endoplasmic reticulum, like ERBP2, and that the two proteins interact in vivo. 15 Loss of ERBP1 from bloodstream-form $T$. brucei initially resulted in a growth defect but 16 proliferation was restored after more prolonged cultivation. Pull-down analysis of tagged ERBP1 17 suggests that it preferentially binds to ribosomal protein mRNAs. The ERBP1 sequence 18 resembles that of Saccharomyces cerevisiae Bfr1, which also localizes to the endoplasmic 19 reticulum and binds to ribosomal protein mRNAs. However, unlike Bfr1, ERBP1 does not bind to mRNAs encoding secreted proteins, and it is also not recruited to stress granules after starvation.
\end{abstract}

\title{
Introduction
}

In Trypanosoma brucei, most regulation of gene expression is post-transcriptional. Protein-coding genes are arranged in polycistronic transcription units, and mRNAs are excised by trans splicing and polyadenylation (Clayton 2019). Levels of constitutively expressed proteins and mRNAs are strongly influenced by codon usage (de Freitas Nascimento et al. 2018; Jeacock et al. 2018), while regulation during development, over the cell cycle, and in response to environmental conditions is effected mainly by RNA-binding proteins. The latter often, but not always, bind sequences in 3'-untranslated regions (3'-UTRs) (Clayton 2019). All mRNAs - whether or not they are subject to specific regulation - are expected to be bound by numerous different proteins, forming a "messenger ribonucleoprotein" (mRNP) assembly.

T. brucei proliferates in mammalian blood and tissue fluids, and in the digestive system of Tsetse flies. The developmental stages that are most accessible to laboratory study are the bloodstream form, which grows at $37^{\circ} \mathrm{C}$ in glucose-rich media and corresponds to the form that grows in mammals, and the procyclic form, which is grown at $27^{\circ} \mathrm{C}$ in proline-rich medium and multiplies in the Tsetse midgut. Purification of mRNPs from bloodstream forms revealed at least 155 proteins that reproducibly could be cross-linked to, and co-purified with, mRNA (Lueong et al. 2016). Although many of the mRNA-binding proteins had recognizable consensus RNA-binding motifs, the list included 49 proteins, including that encoded by Tb927.10.14150, which had no obvious connection to RNA metabolism. Similar studies of Opisthokonts also revealed numerous novel proteins without known RNA-binding domains, which have been named "enigmRBPs" 
41 (Beckmann et al. 2015; Hentze et al. 2018). We will, therefore, call the Tb927.10.14150 protein 42 ERBP1 (for EnigmRBP1).

43 To assess the ability of trypanosome proteins to affect mRNA stability or translation, they were 44 "tethered" to a reporter RNA. To do this, proteins or protein fragments were expressed fused to 45 the $\mathrm{N}$ peptide from bacteriophage lambda. The lambdaN peptide binds an RNA stem-loop called 46 boxB with high affinity. The reporter mRNA encoded a selectable marker with boxB sequences in 47 the 3'-UTR. Proteins were screened first as random fragments (Erben et al. 2014), then at full 48 length (Lueong et al. 2016). Numerous regulators were found, many of which were also in the $49 \mathrm{mRNP}$ proteome (Lueong et al. 2016), and ERBP1 reproducibly conferred a selective advantage 50 when tethered both as fragments and at full length (Erben et al. 2014; Lueong et al. 2016). ERBP1 51 fused C-terminally to GFP was associated with the endoplasmic reticulum in a high-throughput 52 screen of procyclic forms (Dean et al. 2016) and its depletion resulted in a selective disadvantage 53 in a high throughput RNAi screen (Alsford et al. 2005).

54 In this paper, we describe more detailed studies of ERBP1. ERBP1 is predicted to belong to a 55 protein family that is named after a S. cerevisiae protein called Bfr1p (IPR039604 or PTHR31027), 56 with alignment over its entire length. Bfr1p was originally recovered in a screen for high-copy57 number suppression of Brefeldin A toxicity (Jackson \& Kepes 1994). It is an mRNA-binding protein 58 that is associated with polysomes and the endoplasmic reticulum (ER) (Lang et al. 2001; Weidner et al. 2014), and is incorporated into stress granules (Simpson et al. 2014). In yeast, it is associated with over 1000 different mRNAs, enriched for those encoding ribosomal proteins and mRNAs that are translated at the ER (Lapointe et al. 2015). In addition to a role in stress granules, Bfr1 has been implicated in ER quality control (Low et al. 2014) and correct nuclear segregation (Xue et al. 1996). The physical interaction map

64 (https://www.yeastgenome.org/locus/S000005724/interaction) includes five proteins related to mRNA decay: with Xrn1p, Dcp2p, Scp160p, Puf3p, and Asc1p (an orthologue of RACK1 that inhibits translation). Our results reveal that ERBP1 has both similarities with, and differences from, Bfr1.

\section{Materials and Methods}

\section{DNA manipulation and trypanosomes}

Lister 427 strain trypanosomes expressing the tet repressor were used for all experiments, and were cultivated and transfected as described previously (Alibu et al. 2004). All plasmids and oligonucleotides are listed in S3 Table. Expression from tetracycline-inducible promoters was induced with $100 \mathrm{ng} / \mathrm{ml}$ tetracycline, and all growth studies were performed in the absence of selecting antibiotics. Cultures that were used for RNA and protein analysis had a maximum density of $1.5 \times 10^{6} / \mathrm{ml}$.

$783-5 \times 10^{6}$ cells were collected per sample, resuspended in $6 \times$ Laemmli Buffer and heated at $95^{\circ} \mathrm{C}$ 79 for $10 \mathrm{~min}$. The samples were subjected to SDS-PAGE gel electrophoresis using $10 \%$ 80 polyacrylamide gels. The gels were then stained with SERVA blue $\mathrm{G}$ or blotted on a $0.45 \mu \mathrm{m}$ 81 nitrocellulose blotting membrane (Neolabs). To verify the protein transfer, the membrane was 82 stained with Ponceau S (SERVA). The membrane was blocked with 5\% milk in TBS-Tween and 83 incubated with appropriate concentrations of first and secondary antibodies. Western Lightning 84 Ultra (Perkin Elmer) was used as a chemiluminescence system and signals were detected with 
85 the LAS-4000 imager (GE Healthcare) and CCD camera (Fujifilm). Antibodies used were: rabbit

86

87

88

89

90

91

92

93

94

95

96

97

98

99

100

101

102

103

104

105

106

107

108

109

110

111

112

113

114

115

116

117

118

119

120

121

122

123

124

125

126

127

128 anti-Aldolase (1:50000) (Clayton 1987); mouse anti-myc 9E10 (Santa Cruz, 1:200); rabbit Peroxidase anti-Peroxidase (Sigma, 1:20000); rat anti-ribosomal protein S9 (1:1000); antiTrypanothione Reductase (rabbit, gift from L. Krauth-Siegel, BZH Heidelberg); Mouse anti-V5 (Biorad, 1:2000); anti-SCD6 and anti-DHH1 (from S. Kramer, University of Wurzburg, 1:10000 and 1:15000 respectively) and rabbit anti-BiP (from J. Bangs, University of Buffalo, 1:1000).

\section{Digitonin and stress granule fractionation}

For each sample, $3 \times 10^{7}$ cells were collected by centrifugation at $2000 \mathrm{~g}$ for $10 \mathrm{~min}$ at $4^{\circ} \mathrm{C}$. The pellet was resuspended in $100 \mu \mathrm{l} 1 \mathrm{x}$ PBS and centrifuged at $10000 \mathrm{~g}$ for $5 \mathrm{~min}$ at $4^{\circ} \mathrm{C}$. The pellet was then resuspended in $50 \mu \mathrm{l} \mathrm{STE} \mathrm{buffer} \mathrm{(10} \mathrm{mM} \mathrm{Tris-HCl} \mathrm{pH} \mathrm{8.0,} 150 \mathrm{mM} \mathrm{NaCl}, 1 \mathrm{mM}$ EDTA) and centrifuged at $10000 \mathrm{~g}$ for $5 \mathrm{~min}$ at $4^{\circ} \mathrm{C}$. A $10 \mu \mathrm{g} / \mu \mathrm{l}$ digitonin stock solution was heated at $98^{\circ} \mathrm{C}$ for $5 \mathrm{~min}$ and cooled down before use. Seven different digitonin containing solutions, ranging from 0-1.65 $\mu \mathrm{g} / \mathrm{\mu l}$ digitonin, were prepared and each pellet was resuspended properly in $60 \mu \mathrm{l}$ of one solution. The samples were incubated at $25^{\circ} \mathrm{C}$ for $5 \mathrm{~min}$ and then centrifuged immediately at $10000 \mathrm{~g}$ and $4^{\circ} \mathrm{C}$ for $5 \mathrm{~min}$. The supernatant was transferred to another tube containing $20 \mu \mathrm{l} 4 \mathrm{x}$ SDS-PAGE sample buffer. The pellet was washed twice with $1 x$ PBS by centrifugation $\left(4^{\circ} \mathrm{C}\right.$, $10000 \mathrm{~g}, 5 \mathrm{~min}$ ) and finally resuspended in $80 \mu \mathrm{l}$ x Laemmli buffer. Samples were analyzed by Western Blotting. Stress granules were purified exactly as described in (Fritz et al. 2015), and immunoprecipitations were done as described in (Singh et al. 2014).

Immunofluorescence microscopy

Tissue culture glass slides with 8 chambers were treated with $0.1 \%$ Poly-Lysine (Sigma, P-8920). $2.5 \times 10^{6}$ formaldehyde-fixed $T$. brucei were allowed to adhere to poly-lysine-treated chambered slides (Falcon, 354108), permeabilised with $0.2 \%(\mathrm{w} / \mathrm{v})$ Triton X-100 then incubated with proteinspecific antibodies followed fluorescently-labelled second antibodies in PBS containing $0.5 \%$ gelatin. DNA was stained with $100 \mathrm{ng} / \mathrm{ml}$ DAPI (D9542, Sigma-Aldrich). Mitochondria were detected by addition of Mitotracker Red CMXRos ( $50 \mathrm{nM}$, Thermo Fisher Scientific) to the cells 5 min prior to fixation. Images were examined using the Olympus IX81 microscope, 100x Oil objective with a numerical aperture of 1.45. Digital images were taken with ORCA-R2 digital CCD camera C10600 (Hamamatsu) and using the Xcellence RT software. The bright-field images were taken using differential interference contrast (DIC). Fluorescent images were taken as Z-Stacks with a height of roughly $4 \mu \mathrm{m}$ and a step width of $0.2 \mu \mathrm{m}$. The images were deconvoluted (Wiener Filter, Sub-Volume overlap: 20) and then processed using ImageJ. The background was subtracted and brightness and contrast were adjusted automatically. The most in-focus image of the deconvoluted stack was used.

\section{RNA preparation and Northern blotting}

$5 \times 10^{7}$ cells were used for the extraction of total mRNA using peqGold Trifast (PeqLab). RNA was separated on agarose-formaldehyde gels and blotted on a nylon membrane (Amersham Hybond$\mathrm{N}+$, GE Healthcare, RPN203B). RNA was cross-linked on the membrane by UV light $\left(0.2 \mathrm{~J} / \mathrm{cm}^{2}\right)$ and stained afterwards with methylene blue (SERVA) before hybridization with ${ }^{32} \mathrm{P}$-labelled probes, made using either the Prime-IT RmT Random Primer Labelling Kit (Stratagene) or, for oligonucleotides, [ ${ }^{32}$ P]ATP and T4 polynucleotide kinase (New England Biolabs).

\section{Affinity purification and mass spectrometry}

To purify TAP-ERBP1 for mass spectrometry, the protein was subjected to two steps of affinity purification (Estévez et al. 2003). Briefly, the cleared lysate was incubated with IgG sepharose

Peer) reviewing PDF | (2019:08:40114:2:0:NEW 7 Dec 2019) 
129 beads, washed, and then bound proteins were released using TEV protease. The resulting 130 preparation was then allowed to adhere to a calmodulin affinity column, and proteins were eluted 131 with EGTA. Co-purifying proteins from three independent experiments were analyzed by LC/MS 132 by the ZMBH Mass Spectrometry facility. Cell lines expressing TAP-GFP served as control. Raw 133 data were analyzed using MaxQuant 1.5.8.3, with label-free quantification (LFQ), match between 134 runs (between triplicates), and the $\mathrm{iBAQ}$ algorithm enabled. The identified proteins were filtered 135 for known contaminants and reverse hits, as well as hits without unique peptides.

136 Affinity purification and RNASeq

137 For identification of associated RNAs (Droll et al. 2013), $1 \times 10^{9}$ cells the cells were resuspended 138 in $50 \mathrm{ml}$ ice-cold PBS, and UV-crosslinked $\left(2 \times 0.24 \mathrm{~J} / \mathrm{cm}^{2}\right.$, Stratagene UV crosslinker) in two P15 139 Petri dishes on ice. They were then pelleted, snap-frozen and stored in liquid nitrogen before use. 140 The TAP-ERBP1 was allowed to bind to lgG beads, and the unbound lysate was retained as one 141 of the controls. The bound protein was released using TEV protease. Cross-linked proteins were 142 removed by proteinase $\mathrm{K}$ digestion (Droll et al. 2013) and RNA was extracted using peqGold 143 Trifast (peqLab) according to manufacturer's protocol. rRNA was depleted as required, by 144 incubation with complementary oligonucleotides and RNaseH (Minia et al. 2016). The NEBNext 145 Ultra RNA Library Prep Kit for Illumina (New England BioLab) was used for library preparation, 146 prior to sequencing with sequenced at EMBL (HiSeq 2000) to generate 50-base reads. Data were 147 analyzed using an in-house tool (10.5281/zenodo.165132) (Mulindwa et al. 2018).

\section{Results}

\section{ERBP1 is conserved in Trypanosoma}

150 To investigate the function of ERBP1, we first analyzed its sequence. ERBP1 is a 55-kDa

151 protein with an isoelectric point of 5.73. It is predicted (by Phyre2) to consist predominantly of

152 alpha-helices. Homologues are found at the equivalent chromosomal position in other

153 Trypanosoma species, and in Endotrypanum, Paratrypanosoma, Blechomonas and Bodo, but

154 the gene appears to have been lost in Leishmania. The alignment (Supplementary Fig S1) shows that the proteins share extremely acidic C-termini: for example, in T. brucei, the last 33 amino acid residues include 5 aspartates and 14 glutamates, as opposed to 3 lysines. Outside the Kinetoplastida, the nearest matches were not with yeast Bfr1p, but with proteins of unknown function in Galdieria sulphuraria, an acidiphilic red alga, and organisms from the SAR group that probably have incorporated red algal endosymbionts: the oomycete Phytophthora parasitica, the brown alga Ectocarpus siliculosus and the diatom Phaeodactylum tricornutum. The alignment with all these proteins, as well as Bfr1p, showed little sequence identity between the kinetoplastid sequences and all the others (S1 Fig).

\section{ERBP1 is required for normal growth but is not essential} We first confirmed that ERBP1 is indeed required for normal growth of bloodstream-form trypanosomes. To do this, we created bloodstream forms in which one ERBP1 gene was modified to encode a protein with an N-terminal V5 tag (V5-ERBP1). These were transfected with a

168 tetracycline-inducible construct for production of ERBP1 dsRNA. Depletion of ERBP1 clearly inhibited cell proliferation but the cells nevertheless survived (Fig 1). 
170 Since some protein always remains after RNAi, we also tested the ability of the cells to survive in 171 the absence of the protein. Bloodstream form cells without ERBP1 (Fig 2A, B) also grew slower 172 than wild-type (Fig 2C), and this could be compensated by re-expression of the protein (Fig 2D). 173 Cells containing only a single tetracycline-inducible copy of ERBP1-myc (inducible on a knock174 out background, cKO) grew at similar rates to wild-type with or without tetracycline, but the protein 175 was clearly detectable in the absence of induction. The double band from ERBP1 was not always 176 seen but might be caused by acetylation (Moretti et al. 2018) or phosphorylation (Urbaniak et al. 177 2013). Despite these initial results, upon prolonged cultivation, the cells lacking ERBP1 gradually 178 increased their growth rate to wild-type. Survival and recovery of the cells after starvation was 179 also indistinguishable from wild-type (S2 Fig A). In procyclic forms, the endogenous ERBP1 gene 180 could be deleted only if an inducible ERBP1-myc gene was present. Depletion of the ERBP1-myc

181

182

183

184

185

186

187

188

189

190

191

192

194

195

196

197

198

199

200

201

202

203

204

205

206

207

208

209

210

211

212 had no effect on growth or recovery from starvation (S2 Fig B), but some residual ERBP1-myc was doubtless present. It is, therefore, possible that ERBP1 is essential in procyclic forms, but the failure of the deletion could also have been due to a technical problem.

The other high-throughput result that required verification was the effect of ERBP1 when tethered to a reporter mRNA. Full-length ERBP1 had previously been identified as conferring a selective advantage in the screen, suggesting that it could activate expression of the blasticidin resistance marker. It gave no growth advantage in control cells in which the marker mRNA had no boxB sequences, ruling out the possibility that ERBP1 expression by itself results in blasticidin resistance. However, lambdaN-ERBP1 was unable to increase expression of a chloramphenicol acetyltransferase reporter (S3 Fig). We have no explanation of this discrepancy: for both the screen and the individual test, the lambdaN-myc sequence was placed at the $\mathrm{N}$-terminus of the open reading frame.

\section{ERBP1 is associated with the endoplasmic reticulum and with a second ER protein}

To examine the location of ERBP1 in bloodstream-form cells, we examined either $\mathrm{N}$-terminally V5-tagged ERBP1 (V5-ERBP1), or C-terminally myc-tagged ERBP1 (ERBP1-myc) expressed from the endogenous locus (Fig 3 and 4). In both cases, ERBP1 clearly co-localized with the endoplasmic reticulum and to some extent could be found in close proximity to mitochondria and glycosome. We then conducted controlled-digitonin permeabilization studies to evaluate the release of the protein with the same markers (Fig 5). ERBP1 was found to coelute partially with the cytosolic marker but fully with the endoplasmic reticulum marker, suggesting that it is loosely associated with the cytosolic face of the ER.

To investigate the interactions of ERBP1, we integrated a sequence encoding the tandem affinity purification (TAP) tag into the genome such that the tag would be at the ERBP1 N-terminus (TAPERBP1) while the other copy of ERBP1 was deleted. TAP-tagged GFP served as the control. Three proteins reproducibly co-purified with ERBP1: calmodulin, the ATP-dependent RNA helicase HEL67/Vasa-like (Kramer et al. 2012), and a protein of unknown function encoded by Tb927.9.9550 (S1 Table). Calmodulin quite often co-purifies with TAP-tagged proteins (Schimanski et al. 2005). The association with the RNA helicase may be significant; although we have detected it on many other mRNA-related purifications. Tb927.9.9550, however, was a novel protein partner. It is conserved throughout Kinetoplastida, activated in the tethering screen (Erben et al. 2014; Lueong et al. 2016), is in the mRNP proteome (Erben et al. 2014; Lueong et al. 2016), but again lacks any recognizable domains; we, therefore, call it ERBP2. It appeared to be 
213 essential in the high-throughput RNAi screen (Alsford et al. 2005), has a predicted signal peptide 214 and trans-membrane domain, and a GFP-tagged version, like ERBP1, localized to the ER in 215 procyclic form (Dean et al. 2016). To verify the interaction we used bloodstream-form cells 216 containing YFP-in-situ tagged ERBP1, with or without expression of ERBP2-myc. YFP-ERBP1 217 was pulled down with anti-myc antiserum only if ERBP2-myc was present, confirming the 218 interaction (Fig 6A), but the experiment also showed that - at least under the conditions used 219 only a very small proportion of the ERBP1 was associated with ERBP2. This may, therefore, be 220 a transient interaction. ERBP2 is predicted to be predominantly alpha-helical, like ERBP1. The 221 isoelectric point of ERBP2 is 11, so it might interact with the highly acidic ERBP1 C-terminus.

\section{ERBP1 associates with mRNAs encoding ribosomal proteins, and not with starvation 223 stress granules}

224 Finally, we wished to know whether ERBP1 had any mRNA-binding specificity. We, therefore, 225 sequenced mRNAs that were co-purified with TAP-ERBP1 from bloodstream-form cells, 226 comparing them either with one unbound fraction or with the total mRNA. Unusually, the ERBP1 227 mRNA itself was not enriched, as would be expected from pull-down of the nascent polypeptide. 228 However, the two bound fractions clearly separated from all the controls (Fig 6B). Sixty four 229 mRNAs were enriched at least 2-fold in both pull-downs relative to all controls. Strikingly, nearly 230 half of them (29) encode ribosomal proteins. Two glycosomal membrane protein mRNAs, PMP4 231 and PEX11, were also enriched, but those encoding other PEX proteins - including other 232 glycosomal membrane proteins - were not, so the significance of this is uncertain. The length of 233 the associated mRNAs seemed to be important since both ribosomal and non-ribosomal targets 234 are shorter than the median (not shown).

235 It should be noted that the enrichment of mRNAs is calculated relative to the enrichment of other 236 mRNAs, and does not actually tell us which proportion of mRNAs with that sequence is associated 237 with the protein in vivo.

238 In budding yeast, Brf1 is associated with stress granules. To find out whether ERBP1 is stress239 granule associated, we incubated procyclic forms in PBS for 2 hours. Although clear granules 240 containing the marker SCD6 were formed (Fig 7A), V5-ERBP1 remained distributed throughout 241 the cytosol (Fig 7A) and was not associated with the granule fraction (Fig 7B). There is, therefore, 242 no evidence that ERBP1 is involved in the formation of stress granules or survival after starvation.

\section{Discussion}

244 Our results suggest that ERBP1 and its partner ERBP2 may modulate ribosomal expression, and 245 are both associated with the endoplasmic reticulum. Our results revealed both similarities and 246 differences between ERBP1 and Bfr1. Each is associated with the endoplasmic reticulum (Lang 247 et al. 2001), and each binds preferentially to mRNAs encoding ribosomal proteins (Lapointe et al. 248 2015). ERBP1, however, unlike Bfr1, does not show any preference for mRNAs encoding proteins 249 that are imported into the endoplasmic reticulum, and it does not associate with stress granules. 250 The latter observation is consistent with the exclusion of ribosomal protein mRNAs from 251 trypanosome stress granules (Fritz et al. 2015). ERBP1 is also associated with a kinetoplastid252 specific protein, ERBP2 which, like ERBP1, was detected in the mRNP proteome (Lueong et al. 253 2016).

254 Synthesis of ribosomes occupies considerable resources in all growing cells, which leads to a 255 requirement for coordination between ribosomal protein synthesis and rRNA transcription. In 
256 mammalian cells and yeast, this coordination happens in the nucleus, at the level of transcription 257 (Albert et al. 2016; Calo et al. 2015). Trypanosomes also devote considerable resources to 258 ribosome synthesis: in bloodstream-forms, resources devoted to rRNA transcription are at least 259 as great as those needed for the synthesis of mRNAs (Haanstra et al. 2008), and the ribosomal 260 protein mRNAs together constitute more than $10 \%$ of total mRNA (Fadda et al. 2014). Feedback 261 inhibition of rRNA processing is known to occur in trypanosomes upon disruption of ribosome 262 assembly or export (Droll et al. 2010), but coordination of rRNA synthesis with ribosomal protein 263 availability - or vice-versa - has not been investigated. The mRNAs encoding trypanosome 264 ribosomal proteins are longer-lived than most others (Fadda et al. 2014), so at least in the short term, control of their translation might be required in order to respond to altered conditions. Ribosome densities on ribosomal protein mRNAs are significantly lower than on other mRNAs, and most of the open reading frames are also relatively short (median $0.6 \mathrm{~kb}$ ); consequently on average there is only one ribosome per mRNA (Antwi et al. 2016). Low ribosome densities occur if the rate of peptide chain elongation is fast relative to the rate of translation initiation. Ribosomal protein mRNAs indeed have optimal codon usage (de Freitas Nascimento et al. 2018), which would result in rapid elongation, and their short 5'-untranslated regions (Clayton 2019) might assist scanning after $43 \mathrm{~S}$ complex recruitment. These characteristics would lead to high constitutive expression, but would also enable very rapid responses to altered conditions since, at 5-10 residues per second, the single ribosome would run off in less than 1 minute. We, therefore, suggest that several redundant pathways control ribosome protein synthesis, and one of them includes ERBP1.

\section{Conclusions}

280

281

282

283

284

285

286

In Trypanosoma brucei, ribosomal protein encoding mRNAs seem to be excluded from starvation stress granules; however, how granule exclusion of these mRNAs occurs is not known. Our studies suggest that ERBP1 is an RNA-binding protein that associates with ribosomal mRNAs, interacts with ERBP2, localizes to the cytosolic face of the endoplasmic reticulum and it does not associate with stress granules. If ERBP1 is the trans-acting factor needed for the exclusion of these mRNAs from starvation stress granules is still not known. As ERBP1 is required for normal growth but is not essential, we suggest that redundant pathways control ribosome synthesis, and one of them may include ERBP1.

\section{Acknowledgements}

288 We thank Nina Papavisiliou for generously hosting KB during the latter part of this project. We 289 also acknowledge Claudia Helbig and Ute Leibfried for technical support, David Ibberson of the 290 BioQuant sequencing facility for cDNA library construction, and the Mass spectrometry facility of 291 the ZMBH. We thank Luise Krauth-Siegel, (BZH Heidelberg), Susanne Kramer (University of 292 Würzberg) and Jay Bangs (University of Buffalo) for antibodies, and Pia Hartwig for work done 293 during a lab rotation.

\section{References}


296

297

298

299

300

301

302

303

304

305

306

307

308

309

310

311

312

313

314

315

316

317

318

319

320

321

322

323

324

325

326

327

328

329

330

331

332

333

334

335

336

337

338

339

340

341

342

343

344

345

346

Albert B, Knight B, Merwin J, Martin V, Ottoz D, Gloor Y, Bruzzone MJ, Rudner A, and Shore D. 2016. A Molecular Titration System Coordinates Ribosomal Protein Gene Transcription with Ribosomal RNA Synthesis. Mol Cell 64:720-733. 10.1016/j.molcel.2016.10.003

Alibu VP, Storm L, Haile S, Clayton C, and Horn D. 2004. A doubly inducible system for RNA interference and rapid RNAi plasmid construction in Trypanosoma brucei. Mol Biochem Parasitol 139:75-82.

Alsford S, Glover L, and Horn D. 2005. Multiplex analysis of RNA interference defects in Trypanosoma brucei. Mol Biochem Parasitol 139:129-132.

Antwi E, Haanstra J, Ramasamy G, Jensen B, Droll D, Rojas F, Minia I, Terrao M, Mercé C, Matthews K, Myler P, Parsons M, and Clayton C. 2016. Integrative analysis of the Trypanosoma brucei gene expression cascade predicts differential regulation of mRNA processing and unusual control of ribosomal protein expression. BMC Genomics 17:306. 10.1186/s12864-016-2624-3

Beckmann BM, Horos R, Fischer B, Castello A, Eichelbaum K, Alleaume AM, Schwarzl T, Curk T, Foehr S, Huber W, Krijgsveld J, and Hentze MW. 2015. The RNA-binding proteomes from yeast to man harbour conserved enigmRBPs. Nat Commun 6:10127.

Calo E, Flynn RA, Martin L, Spitale RC, Chang HY, and Wysocka J. 2015. RNA helicase DDX21 coordinates transcription and ribosomal RNA processing. Nature 518:249-253. 10.1038 /nature13923

Clayton C. 2019. Control of gene expression in trypanosomatids: living with polycistronic transcription. Royal Society Open Blology 9:190072. 10.1098/rsob.190072

Clayton CE. 1987. Import of fructose bisphosphate aldolase into the glycosomes of Trypanosoma brucei. J Cell Biol 105:2649-2653.

de Freitas Nascimento J, Kelly S, Sunter J, and Carrington M. 2018. Codon choice directs constitutive mRNA levels in trypanosomes. Elife 7. 10.7554/eLife.32467

Dean S, Sunter J, and Wheeler R. 2016. TrypTag.org: A trypanosome genome-wide protein localisation resource. Trends Parasitol 33:80-82.

Droll D, Archer S, Fenn K, Delhi P, Matthews K, and Clayton C. 2010. The trypanosome Pumilio-domain protein PUF7 associates with a nuclear cyclophilin and is involved in ribosomal RNA maturation. FEBS Lett 84:1156-1162. 10.1016/j.febslet.2010.02.018

Droll D, Minia I, Fadda A, Singh A, Stewart M, Queiroz R, and Clayton C. 2013. Posttranscriptional regulation of the trypanosome heat shock response by a zinc finger protein. PLoS Pathog 9:e1003286.

Erben E, Fadda A, Lueong S, Hoheisel J, and Clayton C. 2014. Genome-wide discovery of post-transcriptional regulators in Trypanosoma brucei. PLoS Pathog 10:e1004178. doi:10.1371/journal.ppat.1004178

Estévez AM, Lehner B, Sanderson CM, Ruppert T, and Clayton C. 2003. The roles of intersubunit interactions in exosome stability. $J$ Biol Chem 278:34943-34951.

Fadda A, Ryten M, Droll D, Rojas F, Färber V, Haanstra J, Bakker B, Matthews K, and Clayton C. 2014. Transcriptome-wide analysis of mRNA decay reveals complex degradation kinetics and suggests a role for co-transcriptional degradation in determining mRNA levels. Mol Microbiol 94:307-326. 10.1111/mmi.12764

Fritz M, Vanselow J, Sauer N, Lamer S, Goos C, Siegel T, Subota I, Schlosser A, Carrington M, and Kramer S. 2015. Novel insights into RNP granules by employing the trypanosome's microtubule skeleton as a molecular sieve. Nucleic Acids Res 43:8013-8032.

Haanstra J, Stewart M, Luu V-D, van Tuijl A, Westerhoff H, Clayton C, and Bakker B. 2008. Control and regulation of gene expression: quantitative analysis of the expression of phosphoglycerate kinase in bloodstream form Trypanosoma brucei. J Biol Chem 283:2495-2507.

Hentze MW, Castello A, Schwarzl T, and Preiss T. 2018. A brave new world of RNA-binding proteins. Nat Rev Mol Cell Biol. 10.1038/nrm.2017.130

Peer) reviewing PDF | (2019:08:40114:2:0:NEW 7 Dec 2019) 
347

348

349

350

351

352

353

354

355

356

357

358

359

360

361

362

363

364

365

366

367

368

369

370

371

372

373

374

375

376

377

378

379

380

381

382

383

384

385

386

387

388

389

390

391

392

393

394

395

396

Jackson CL, and Kepes F. 1994. BFR1, a multicopy suppressor of brefeldin A-induced lethality, is implicated in secretion and nuclear segregation in Saccharomyces cerevisiae. Genetics 137:423-437.

Jeacock L, Faria J, and Horn D. 2018. Codon usage bias controls mRNA and protein abundance in trypanosomatids. Elife 7. 10.7554/eLife.32496

Kramer S, Marnef A, Standart N, and Carrington M. 2012. Inhibition of mRNA maturation in trypanosomes causes the formation of novel foci at the nuclear periphery containing cytoplasmic regulators of mRNA fate. J Cell Sci 125:2896-2909. 10.1242/jcs.099275

Lang BD, Li A, Black-Brewster HD, and Fridovich-Keil JL. 2001. The brefeldin A resistance protein Bfr1p is a component of polyribosome-associated mRNP complexes in yeast. Nucleic Acids Res 29:2567-2574. 10.1093/nar/29.12.2567

Lapointe CP, Wilinski D, Saunders HA, and Wickens M. 2015. Protein-RNA networks revealed through covalent RNA marks. Nat Methods 12:1163-1170. 10.1038/nmeth.3651

Low YS, Bircham PW, Maass DR, and Atkinson PH. 2014. Kinetochore genes are required to fully activate secretory pathway expansion in S. cerevisiae under induced ER stress. Mol Biosyst 10:1790-1802. 10.1039/c3mb70414a

Lueong S, Merce C, Fischer B, Hoheisel J, and Erben E. 2016. Gene expression regulatory networks in Trypanosoma brucei: insights into the role of the mRNA-binding proteome. Mol Microbiol 100:457-471.

Minia I, Merce C, Terrao M, and Clayton C. 2016. Translation regulation and RNA granule formation after heat shock of procyclic form Trypanosoma brucei: many heat-induced mRNAs are increased during differentiation to mammalian-infective forms. PLoS Negl Trop Dis 10:e0004982.

Moretti NS, Cestari I, Anupama A, Stuart K, and Schenkman S. 2018. Comparative Proteomic Analysis of Lysine Acetylation in Trypanosomes. J Proteome Res 17:374-385. 10.1021/acs.jproteome.7b00603

Mulindwa J, Leiss K, Ibberson D, Kamanyi Marucha K, Helbig C, Melo do Nascimento L, Silvester E, Matthews K, Matovu E, Enyaru J, and Clayton C. 2018. Transcriptomes of Trypanosoma brucei rhodesiense from sleeping sickness patients, rodents and culture: Effects of strain, growth conditions and RNA preparation methods. PLoS Negl Trop Dis 12:e0006280. 10.1371/journal.pntd.0006280

Schimanski B, Nguyen T, and Günzl A. 2005. Highly efficient tandem affinity purification of trypanosome protein complexes based on a novel epitope combination. Eukaryot Cell 4:1942-1950.

Simpson CE, Lui J, Kershaw CJ, Sims PF, and Ashe MP. 2014. mRNA localization to P-bodies in yeast is bi-phasic with many mRNAs captured in a late Bfr1p-dependent wave. J Cell Sci 127:1254-1262. 10.1242/jcs.139055

Singh A, Minia I, Droll D, Fadda A, Clayton C, and Erben E. 2014. Trypanosome MKT1 and the RNA-binding protein $\mathrm{ZC} 3 \mathrm{H} 11$ : interactions and potential roles in post-transcriptional regulatory networks. Nucleic Acids Res 42:4652-4668. 10.1093/nar/gkt1416

Urbaniak MD, Martin D, and Ferguson MA. 2013. Global quantitative SILAC phosphoproteomics reveals differential phosphorylation is widespread between the procyclic and bloodstream form lifecycle stages of Trypanosoma brucei. J Proteome Res 12:22332244. 10.1021/pr400086y

Weidner J, Wang C, Prescianotto-Baschong C, Estrada AF, and Spang A. 2014. The polysomeassociated proteins Scp160 and Bfr1 prevent $P$ body formation under normal growth conditions. J Cell Sci 127:1992-2004. 10.1242/jcs.142083

Xue Z, Shan X, Sinelnikov A, and Melese T. 1996. Yeast mutants that produce a novel type of ascus containing asci instead of spores. Genetics 144:979-989.

Peer) reviewing PDF | (2019:08:40114:2:0:NEW 7 Dec 2019) 
Figure 1

ERBP1 is required for normal growth of bloodstream-form T. brucei.

Bloodstream forms in which one ERBP1 gene was V5-tagged in situ at the 5'-end were used, with inducible expression of stem-loop ERBP1 dsRNA. A. Depletion of V5-ERBP1 after RNAi.

$3 \times 10^{6}$ cells were collected each day and V5-ERBP1 was detected by Western Blotting.

Aldolase was used as loading control. Numbers below the blot indicate the quantification of the V5-ERBP1 signals normalized to the uninduced RNAi at day 0. B. Growth curve for three Tet+ (red) and Tet- (grey) clones analysed in three independent experiments. C. Doubling times (in h) for the 3 experiments are shown.

A
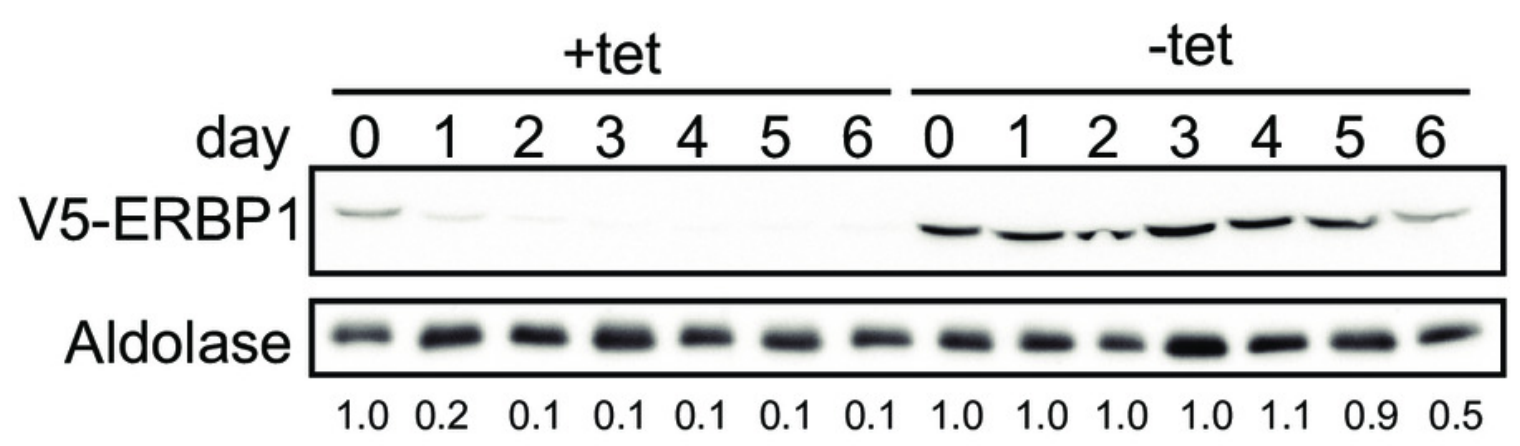

B

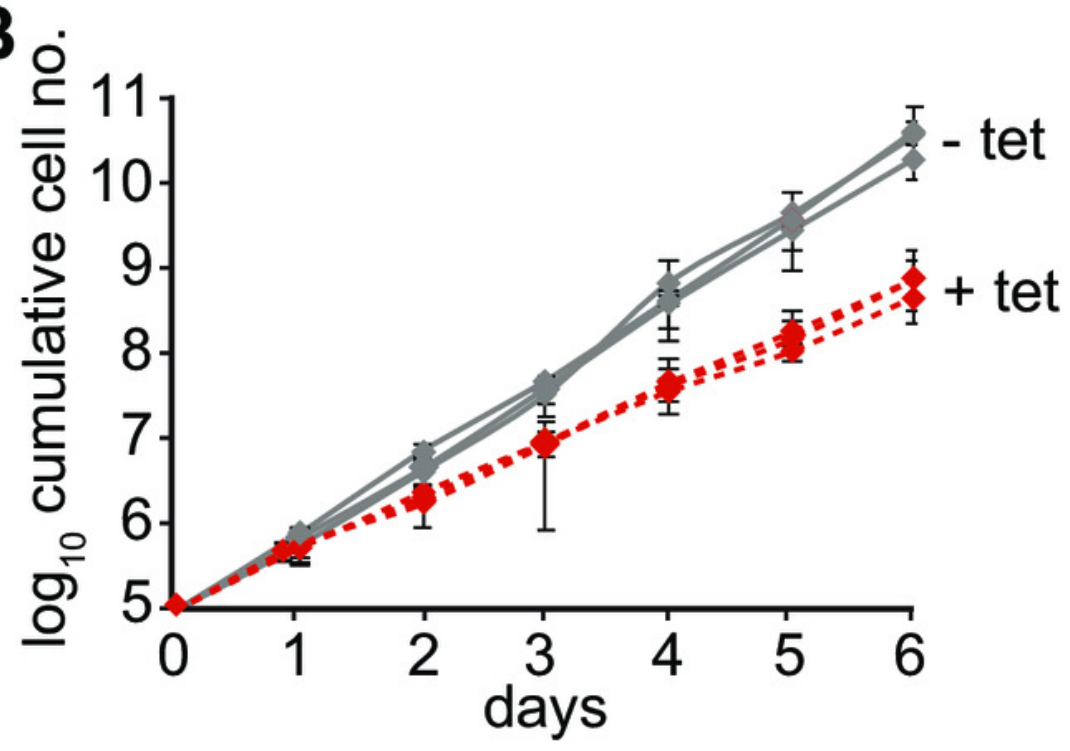

C

doubling time (h)
\begin{tabular}{|r|r|r|r|}
\hline & \multicolumn{1}{|c|}{ A } & \multicolumn{1}{c|}{ B } & \multicolumn{1}{c|}{ C } \\
\hline -tet & 7.8 & 7.6 & 8.1 \\
+tet & 11.2 & 11.4 & 12.1 \\
\hline
\end{tabular}




\section{Figure 2}

Bloodstream-form trypanosomes survive without ERBP1.

A. Schematic representation of the ERBP1 gene before and after replacement with selectable marker genes. Primers for PCR and corresponding product lengths (in $\mathrm{kb}$ ) are shown. B. DNA from wild type (wt) and double knock-out (dKO) cells was analysed by PCR using the primers shown in (A). The primers used are indicated above each image. C. Cumulative growth curve for 3 independent experiments, each with three different clones for wild-type (wt), single knock-out (sKO) and double knock-out (dKO). Error bars indicate standard deviation from results for 3 clones; each was measured 3 times then for each, and average was taken. D. Expression of ERBP1-myc complements the defect in dKO cells. The dKO cells were complemented with tetracycline-inducible ERBP1-myc (complemented KO; cKO). The graph shows the cumulative parasite numbers. E. Western blot from (D) using $3 \times 10^{6}$ cells/lane. Some ERBP1-myc is detectable in the absence of tetracycline inducer. F. As (D) but for procyclic forms. As homozygous gene replacement failed in procyclic forms, the sKO cells were complemented with tetracycline-inducible ERBP1-myc. G. Western blot from (F) using $3 \times 10^{6}$ cells/lane. Aldolase was used as loading control. 
A

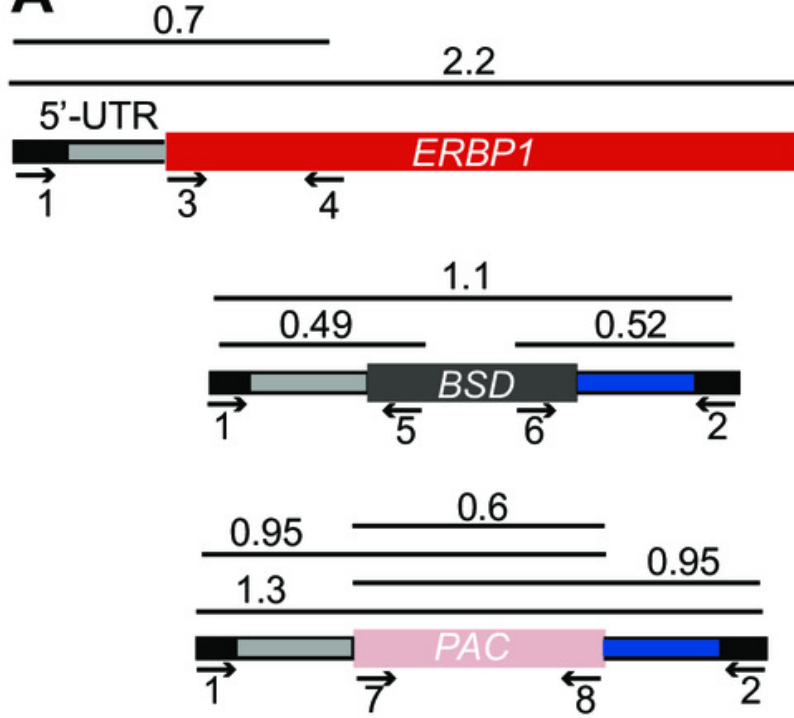

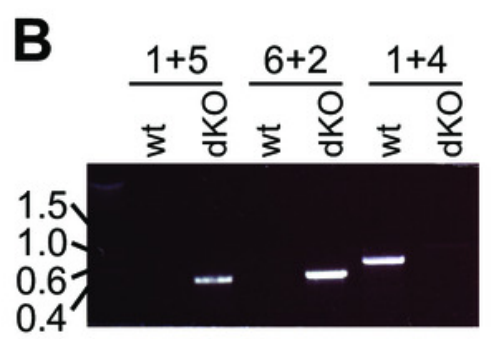

$\mathrm{kb}$

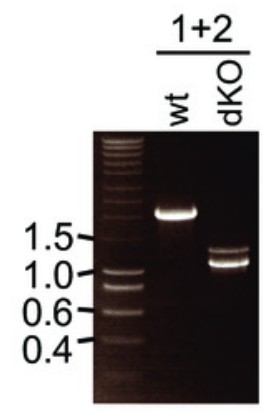

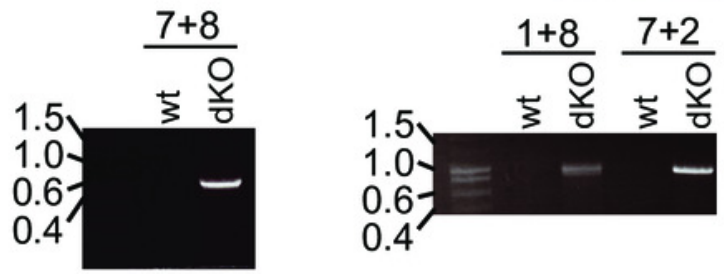

C

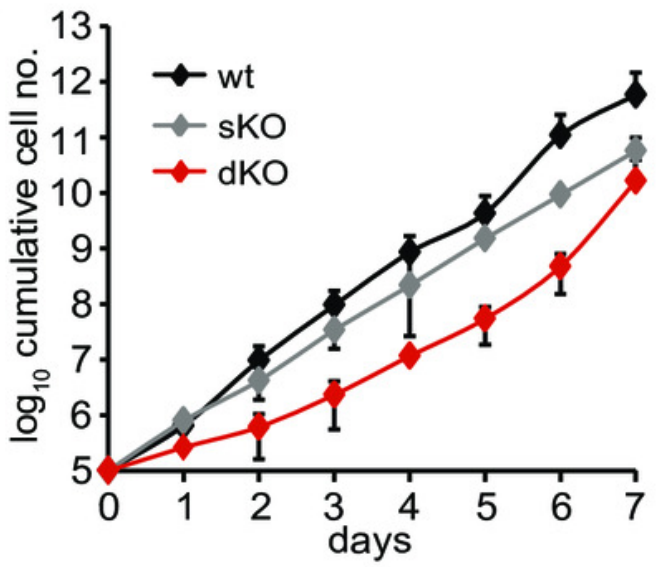

$\mathbf{F}$

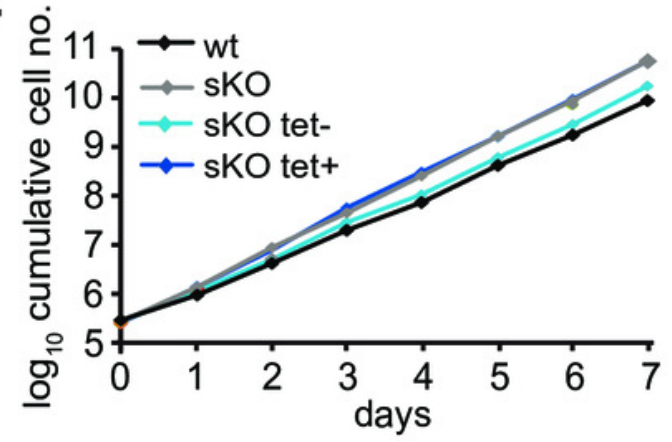

3'-UTR

๘

D

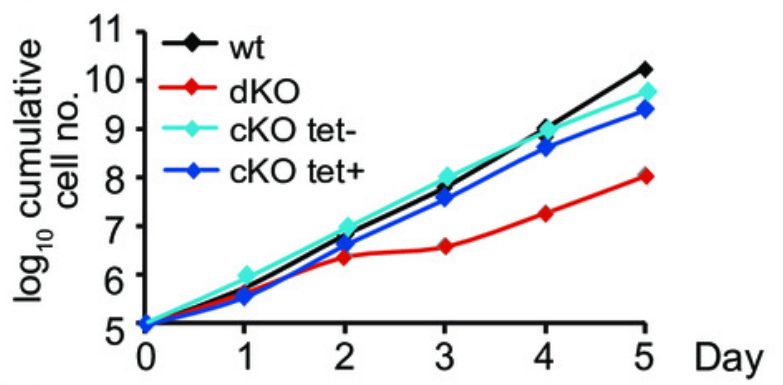

E

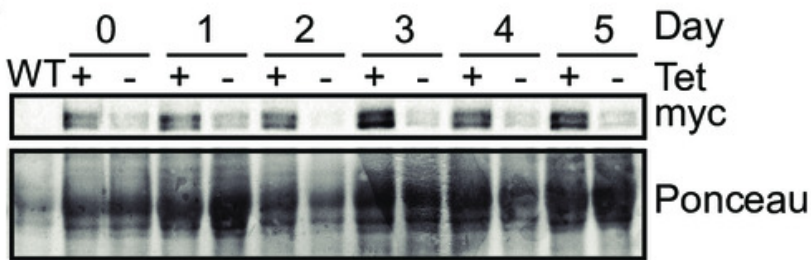

G sKO tet+

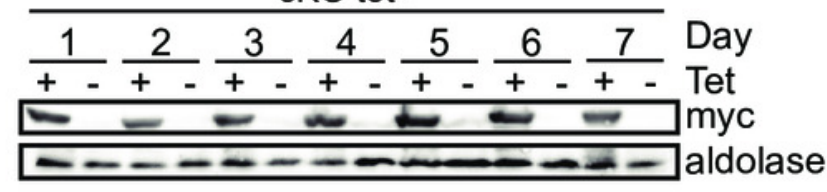




\section{Figure 3}

V5-ERBP1 colocalises with the endoplasmic reticulum.

Bloodstream forms expressing N-terminally V5-tagged ERBP from the endogenous locus (V5ERBP1) were examined by immunofluorescence microscopy. Cells without V5 served as control. Nuclear and kinetoplast DNA was stained with DAPI (blue). Representative images are shown for three independent experiments. Z-stacks were examined using the Olympus CellR microscope and $100 \times$ magnification, and Images were deconvoluted. Scale bar: $10 \mu \mathrm{m}$. A. V5-ERBP1 is red and cytosolic Trypanothione Reductase (=TR) is green. B. V5-ERBP1 is red and glycosomal aldolase is green. C. V5-ERBP1 is green and mitotracker is red D. V5ERBP1 is red and the endoplasmic reticulum marker BiP is green. 


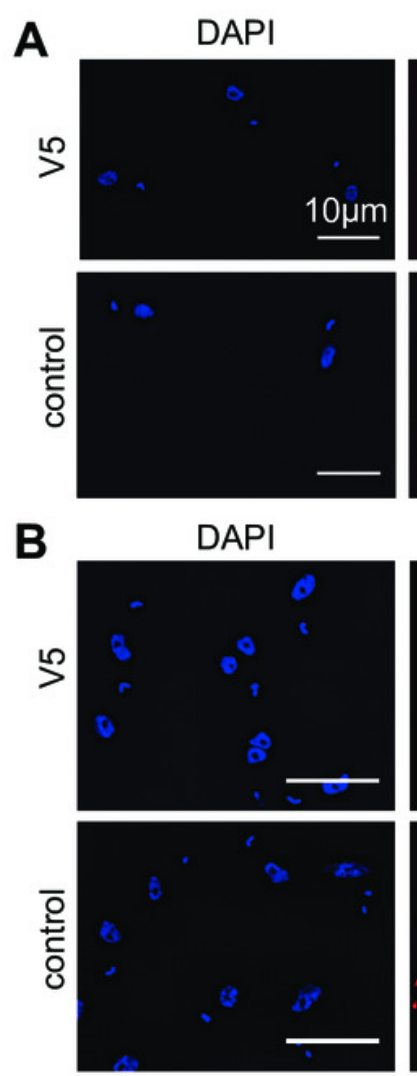

V5-ERBP1

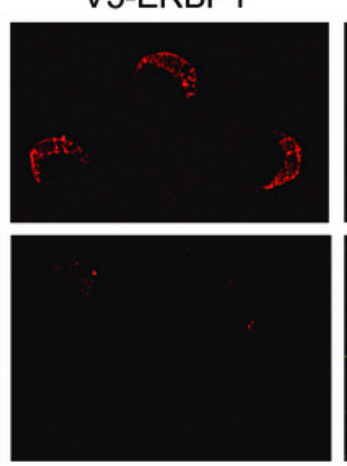

V5-ERBP1
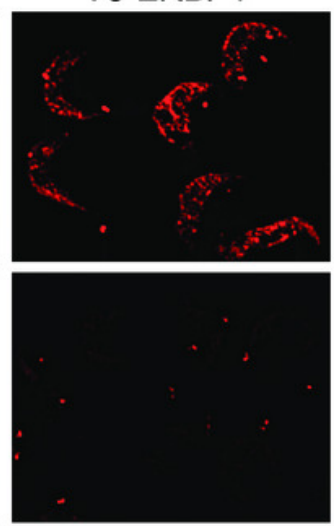

C
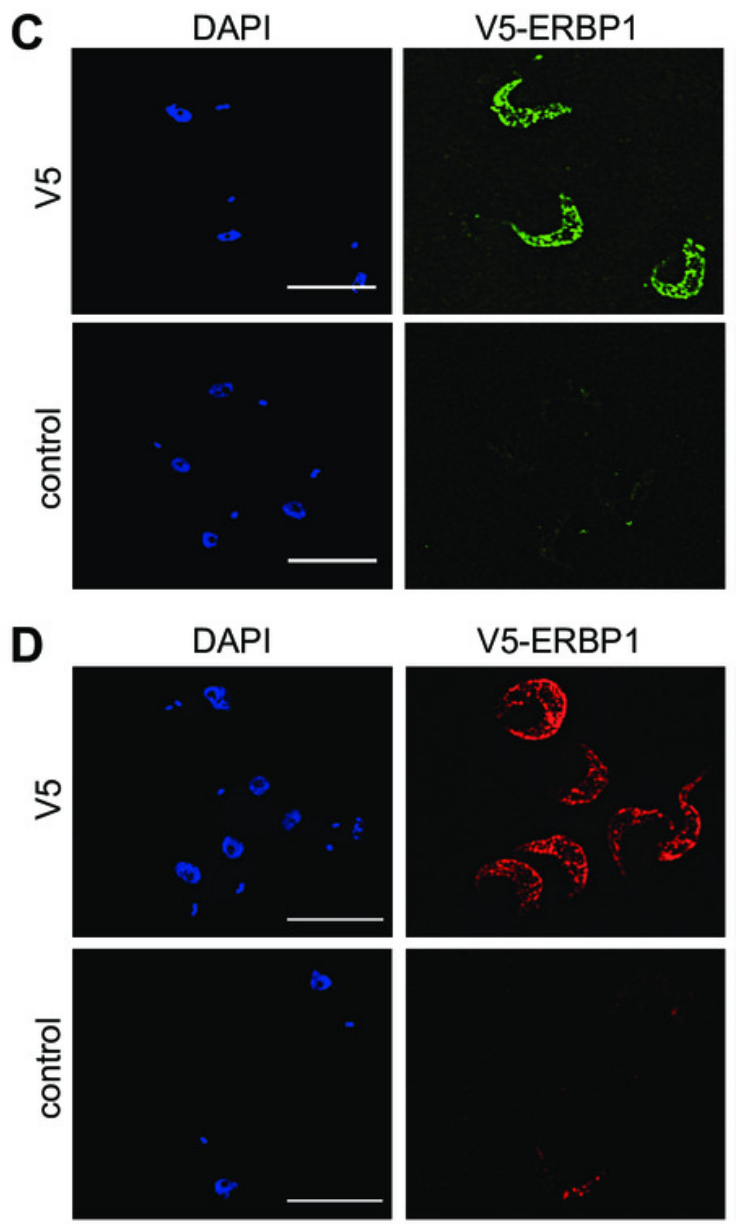
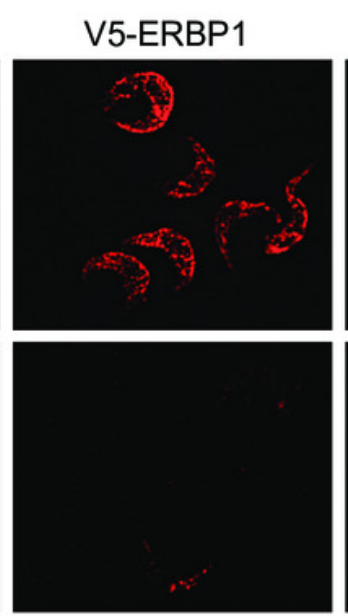

TR

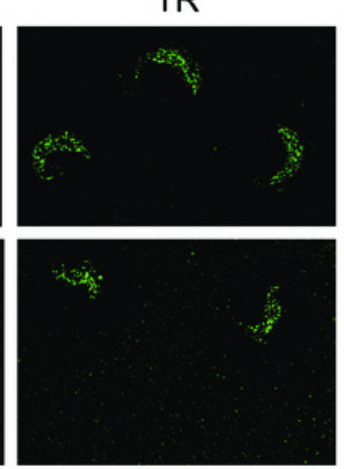

Aldolase
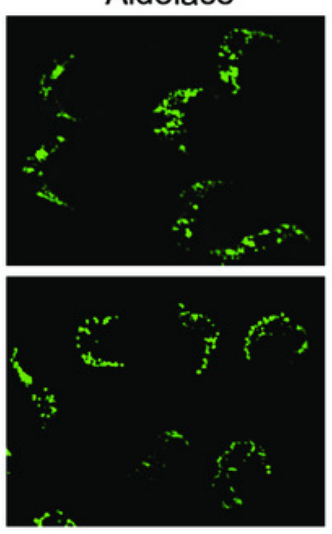

Mitotracker
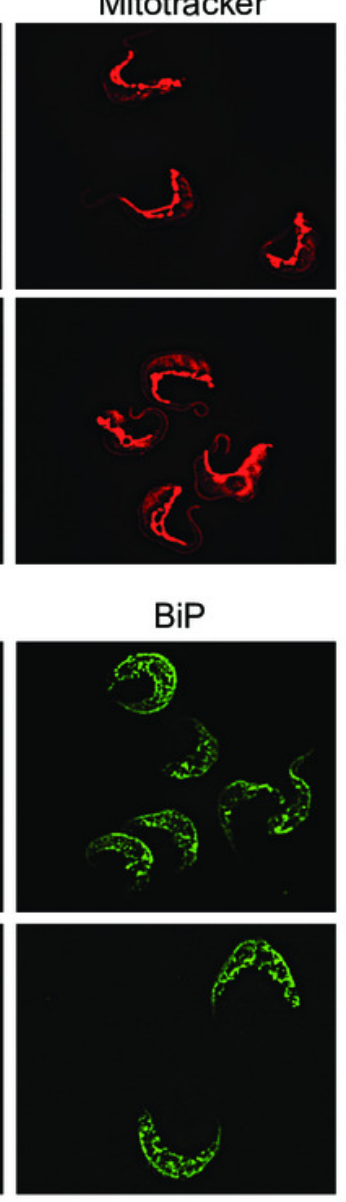

merge

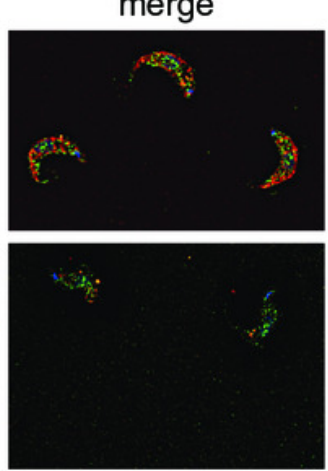

merge
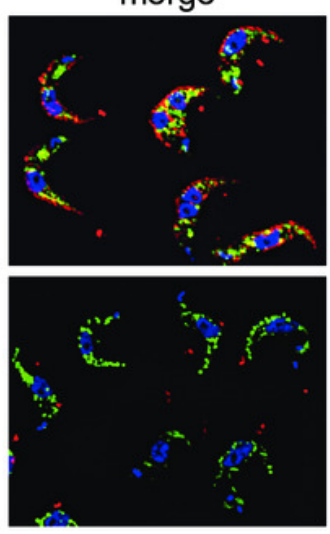

merge
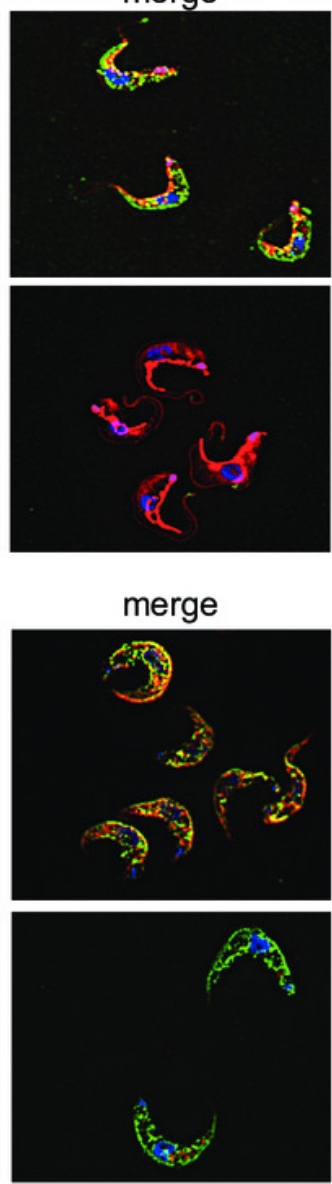

DIC

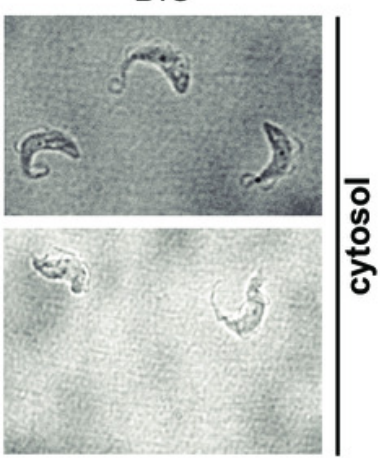

DIC

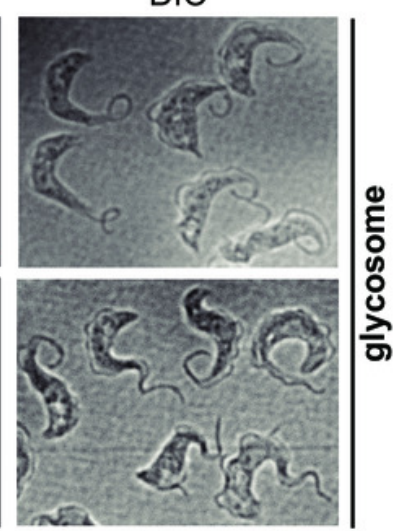

DIC

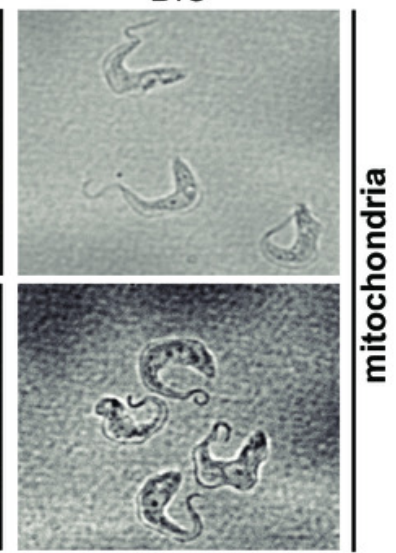

DIC

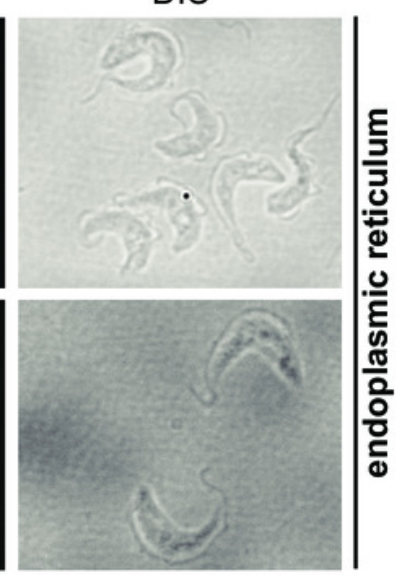




\section{Figure 4}

ERBP1-myc colocalises with the endoplasmic reticulum.

Bloodstream forms expressing C-terminally myc-tagged ERBP from the endogenous locus (ERBP1-myc) were examined by immunofluorescence microscopy. Cells without myc served as control. Nuclear and kinetoplast DNA was stained with DAPI (blue). Representative images are shown for three independent experiments. Z-stacks were examined using the Olympus CellR microscope and $100 \times$ magnification, and Images were deconvoluted. Scale bar: $10 \mu \mathrm{m}$. A. ERBP1-myc is red and cytosolic Trypanothione Reductase (TR) is green. B. ERBP1-myc is red and glycosomal aldolase is green. C. ERBP1-myc is green and mitotracker is red $\mathbf{D}$. ERBP1-myc is red and the endoplasmic reticulum marker BiP is green. 
A
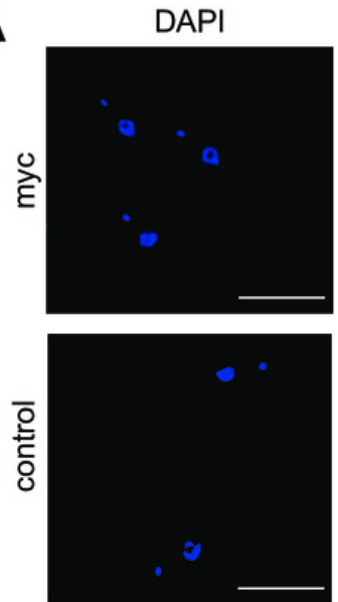

B
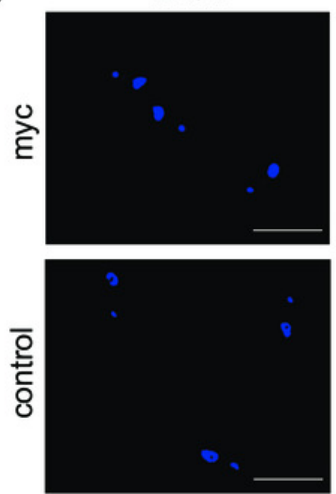

C
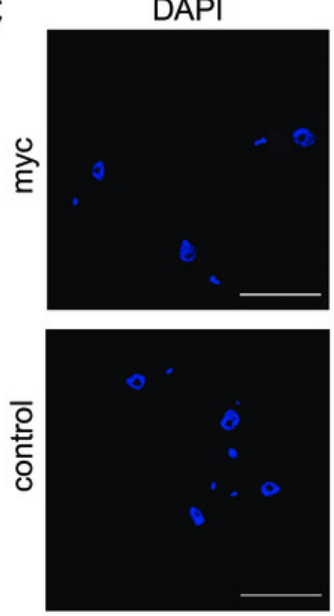

D

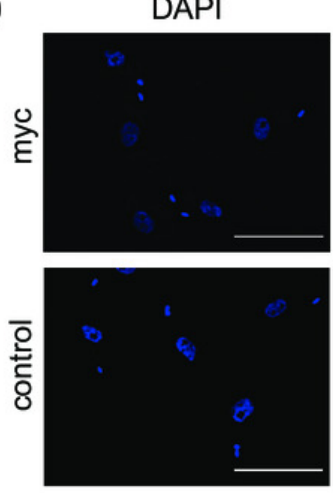

ERBP1-myc
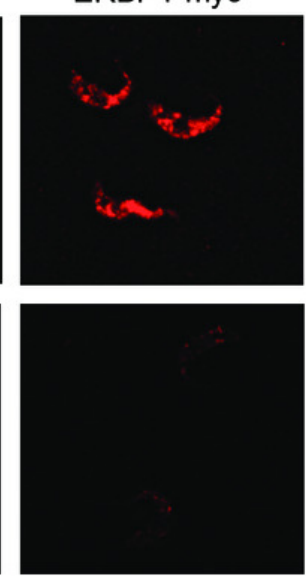

ERBP1-myc
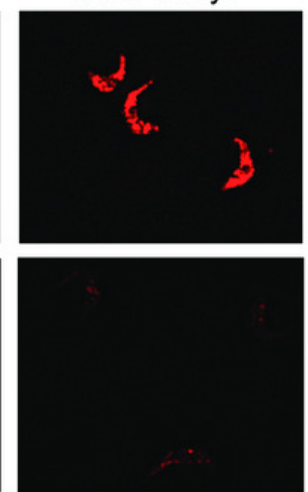

ERBP1-myc
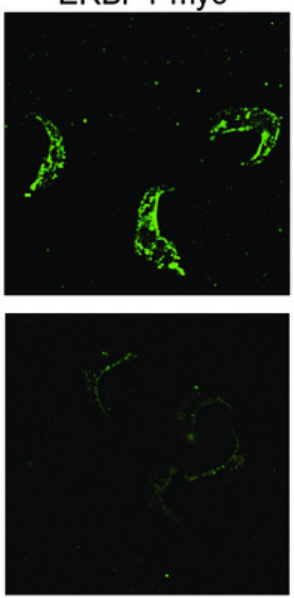

ERBP1-myc
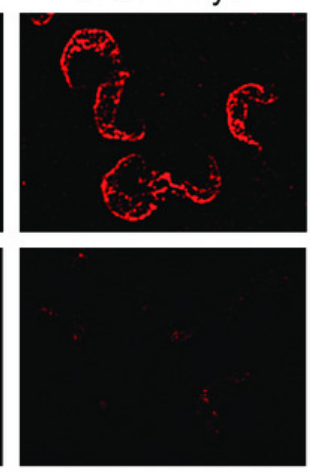
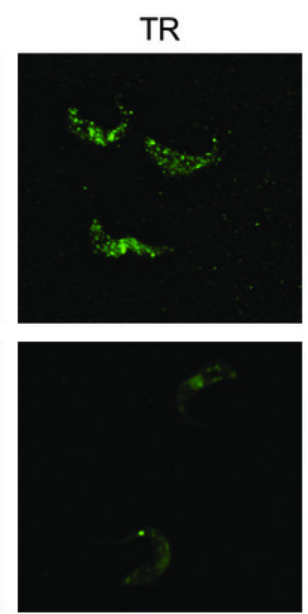

Aldolase
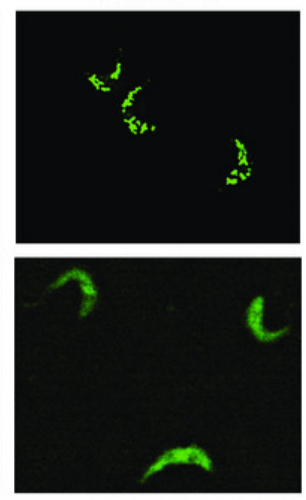

Mitotracker
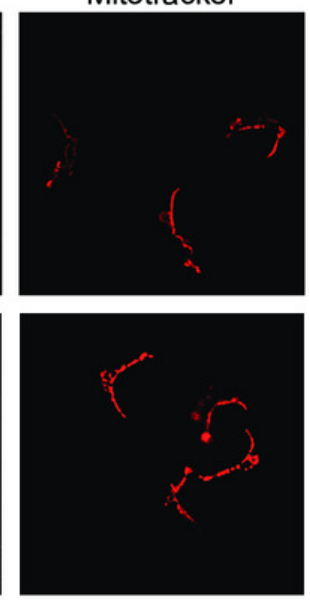

BiP
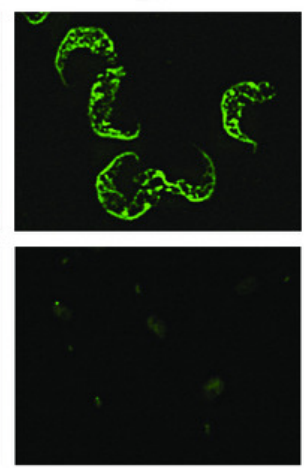
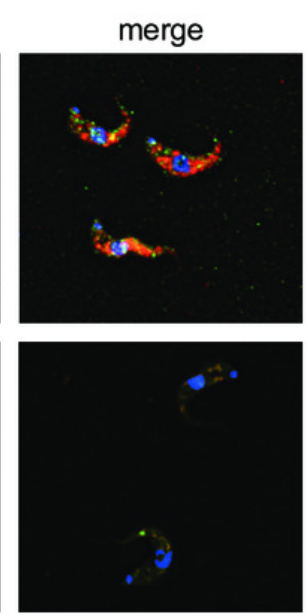

merge
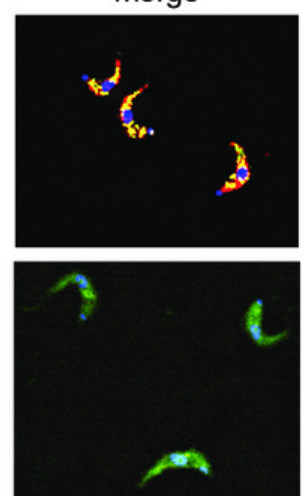

merge
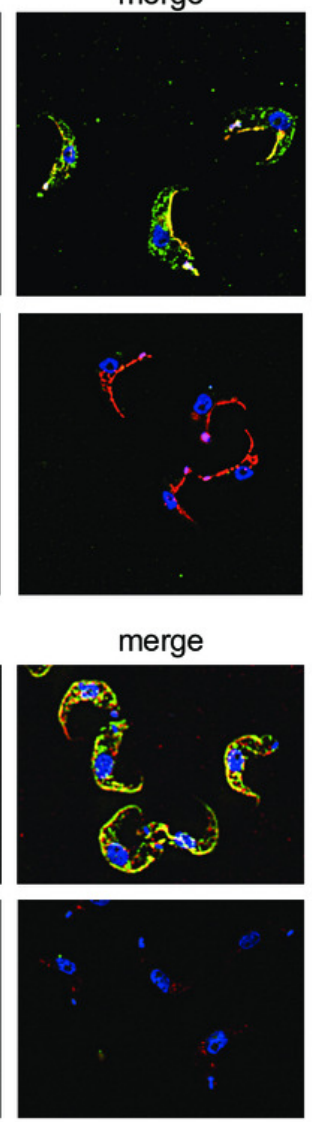

옹

हี

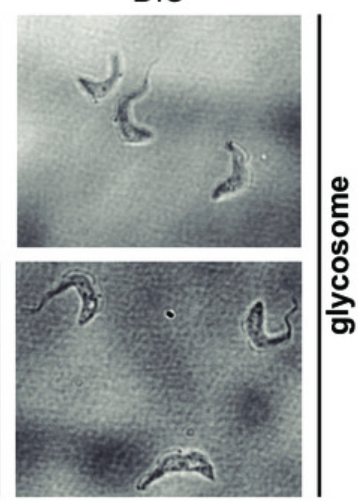

DIC

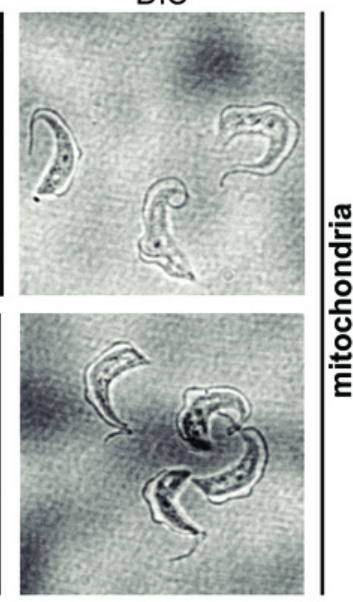

DIC

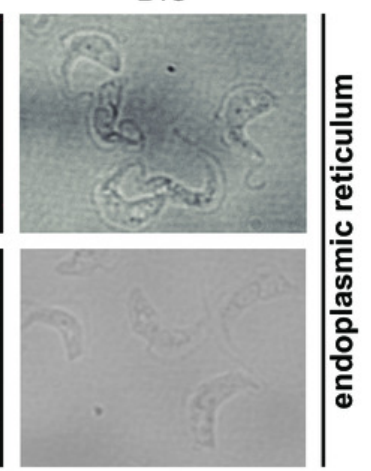

Peer] reviewing PDF | (2019:08:40114:2:0:NEW 7 Dec 2019) 


\section{Figure 5}

ERBP1 is not inside a membrane-bound compartment.

Bloodstream-form cells expressing V5-ERBP1 (A) or ERBP1-myc (B) were treated with increasing digitonin:protein (mg:mg) ratios as indicated at the top. Samples were centrifuged and the supernatant and pellet were analysed by Western Blotting. Numbers below the blots indicate quantification of the corresponding signal. Aldolase (glycosome); TR: Trypanothione Reductase (cytosol); LipDH: Lipoamide Dehydrogenase (mitochondria); BiP: Binding Immunoglobulin Protein (ER).

A

V5-ERBP1

digitionin:protein ratio
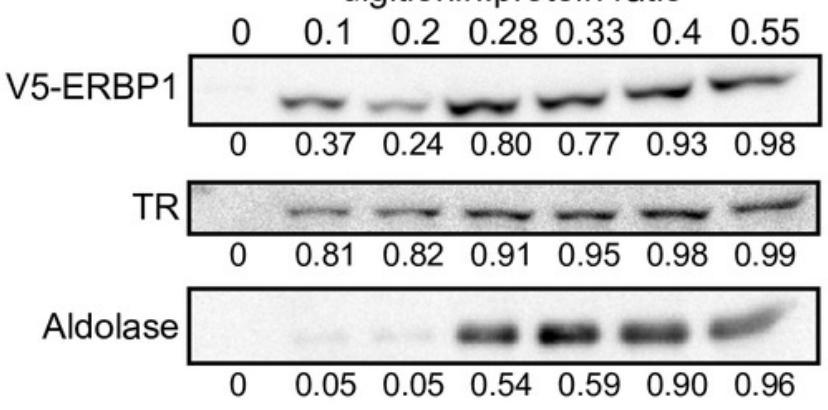

LipDH

$\begin{array}{llllllll}0 & 0.09 & 0.10 & 0.29 & 0.24 & 0.27 & 0.58\end{array}$

V5-ERBP1
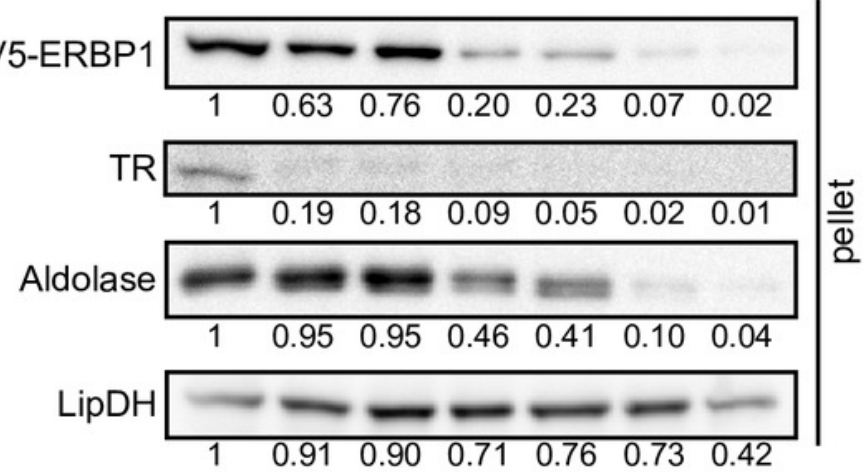

B

ERBP1-myc

digitionin:protein ratio

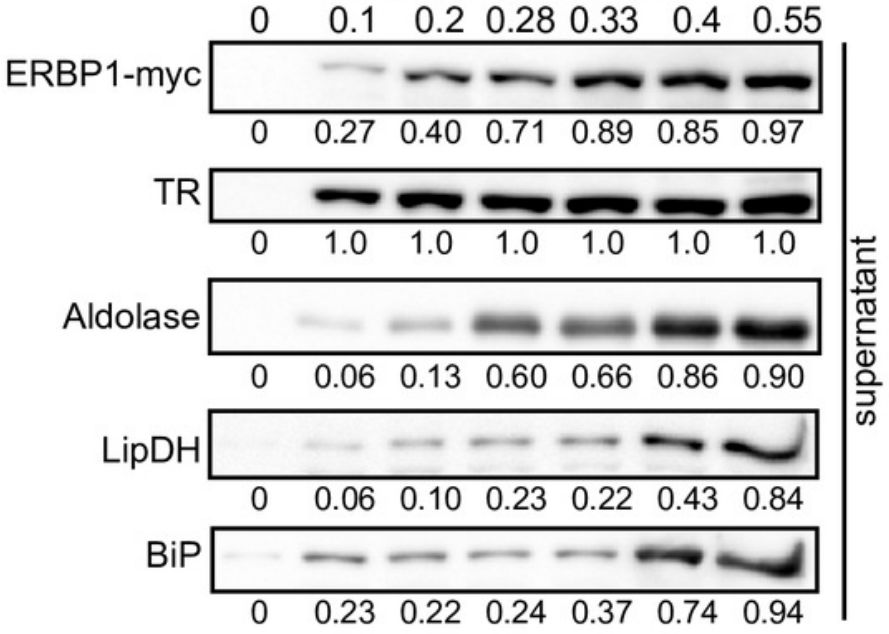

ERBP1-myc

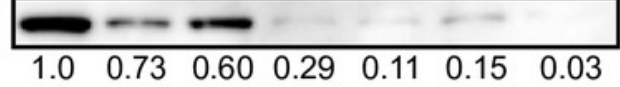

$$
\text { TR }
$$

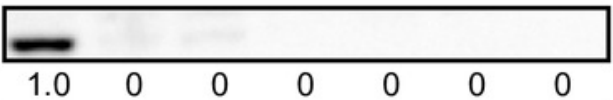

Aldolase
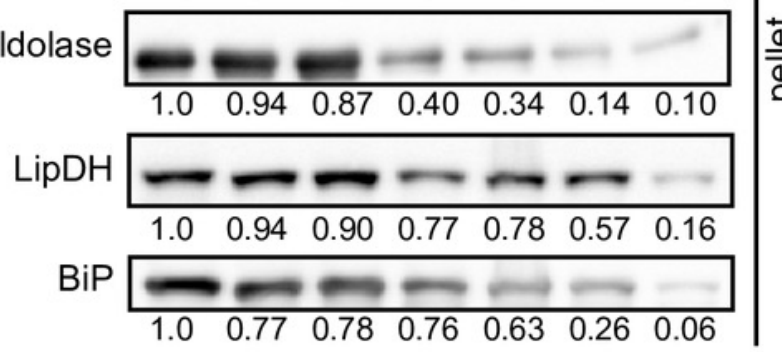


\section{Figure 6}

Interactions of ERBP1.

A. ERBP2 co-precipitates with ERBP1. Extracts were made from cells expressing YFP-ERBP1, with or without ERBP2-myc. After immunoprecipitation with anti-myc, a Western blot was probed for myc and for YFP as indicated. The stained membrane is shown below. I = input; $U B=$ unbound fraction; $E=$ eluate from anti-myc immunoprecipitation. B. Principal component analysis of transcriptomes after RNA-Seq of total mRNA (WT) and after pull-down of TAP-ERBP1; UB = unbound; $B=$ bound (eluate from the affinity matrix). C. Functions of proteins encoded by ERBP1-associated mRNAs. The pie chart show the distributions for mRNAs that were at least 2-fold enriched in both bound fractions relative to the controls. Details are in supplementary Table S2. 
A

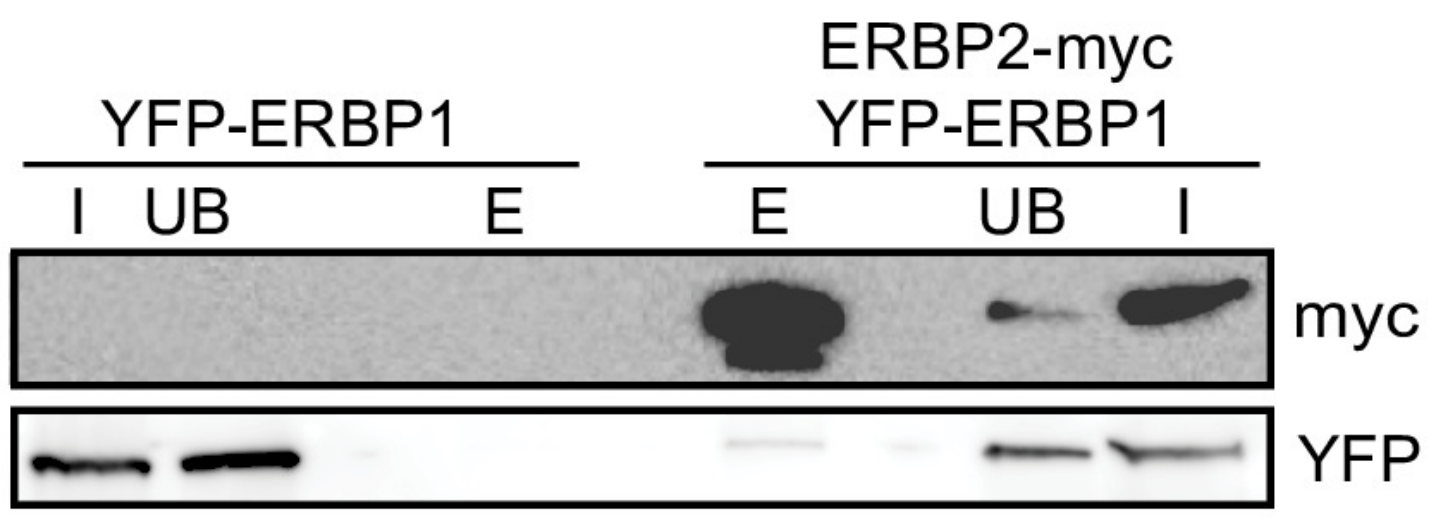

B

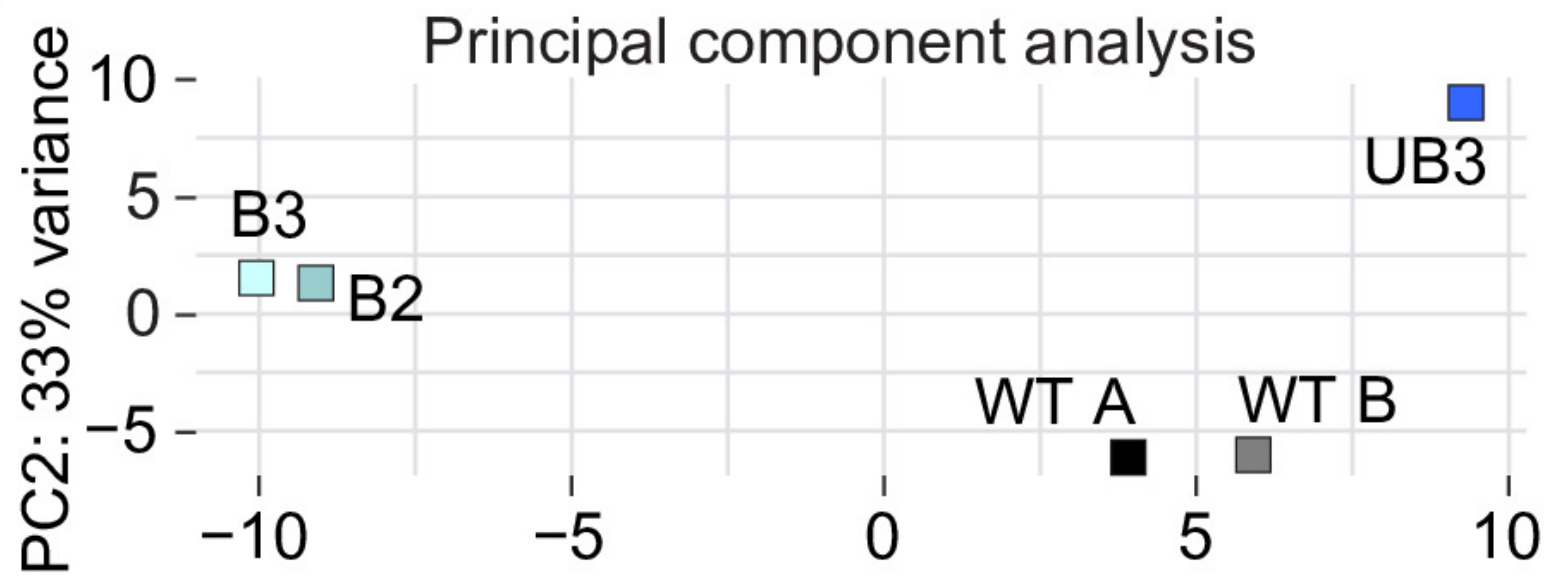

PC1: $64 \%$ variance

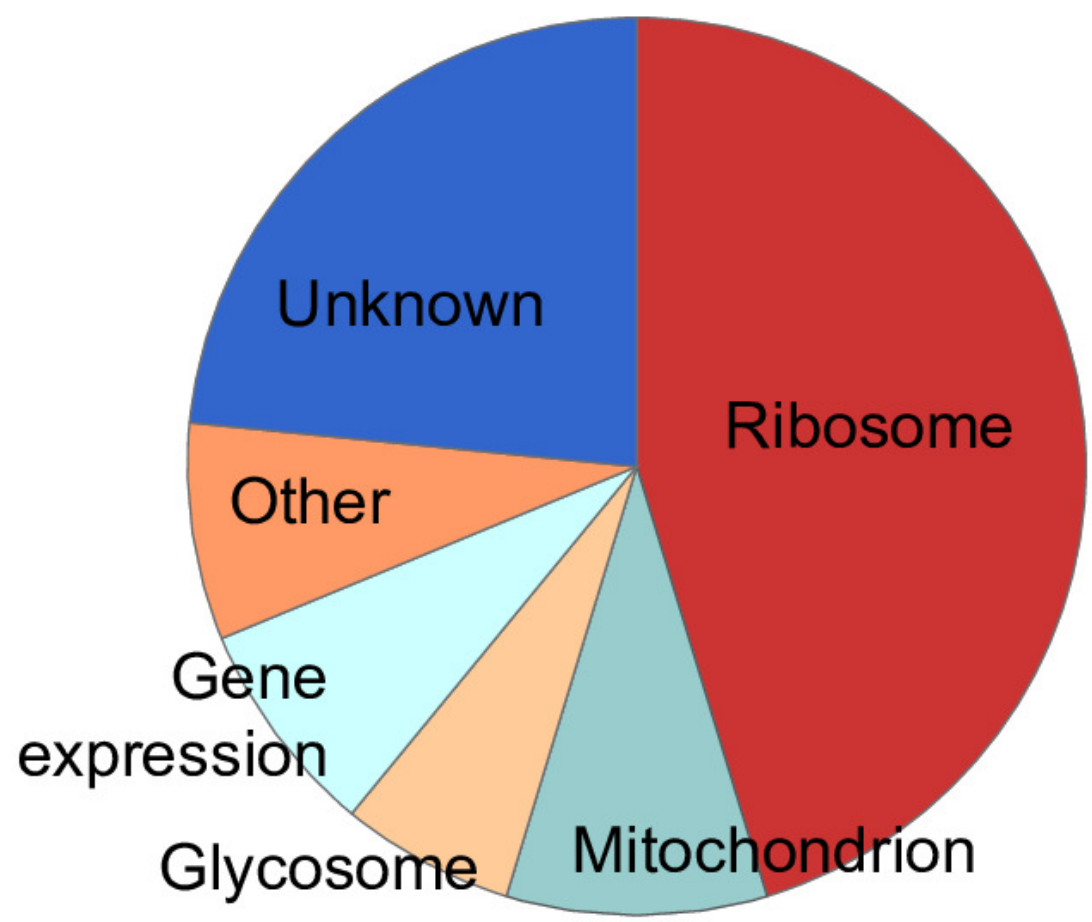




\section{Figure 7}

V5-ERBP1 is not concentrated in starvation stress granules.

A. Procyclic forms expressing V5-ERBP1 were incubated for $2 \mathrm{~h}$ in $1 \times$ PBS, then fixed and stained for V5 (red) and the stress granule marker SCD6 (green). Nuclear and kinetoplast DNA were stained with DAPI (blue). Z-stacks were examined using the Olympus CellR microscope and $100 \times$ magnification. Images were deconvoluted. The control was not starved in PBS. B. V5-ERBP1-expressing procyclic forms were starved as in (A) and then fractionated to concentrate stress granules.
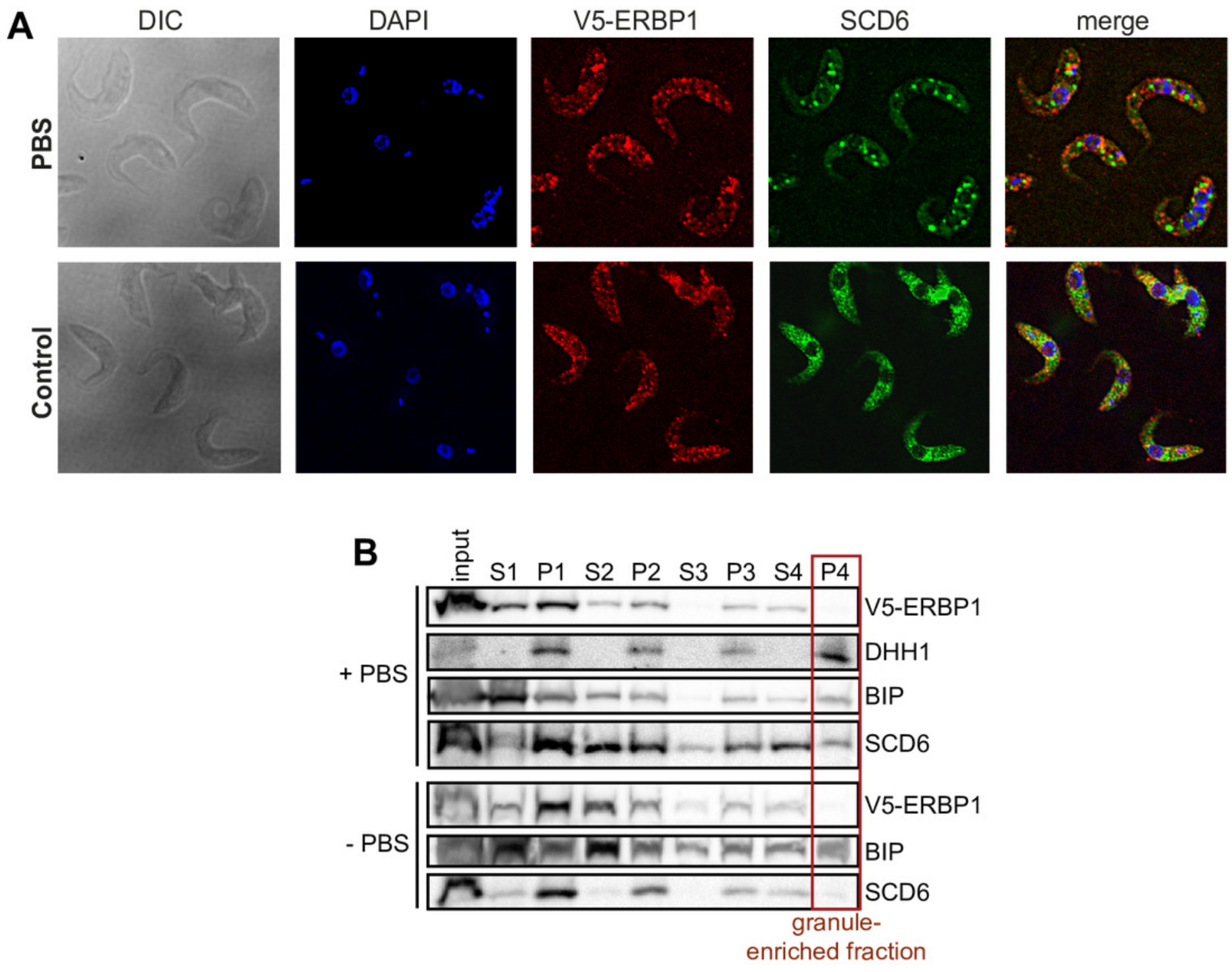